\title{
Oxygen-vacancy-induced local ferromagnetism as a driving mechanism in enhancing the magnetic response of ferrites
}

\author{
C. E. Rodríguez Torres, ${ }^{1,}{ }^{*}$ G. A. Pasquevich, ${ }^{1}$ P. Mendoza Zélis, ${ }^{1}$ F. Golmar, ${ }^{2}$ S. P. Heluani, ${ }^{3}$ Sanjeev K. Nayak, ${ }^{4}$ \\ Waheed A. Adeagbo, ${ }^{4}$ Wolfram Hergert, ${ }^{4}$ Martin Hoffmann, ${ }^{4,5}$ Arthur Ernst, ${ }^{5}$ P. Esquinazi, ${ }^{6}$ and S. J. Stewart ${ }^{1, \dagger}$ \\ ${ }^{1}$ IFLP-CCT-La Plata-CONICET and Departamento de Física, Facultad de Ciencias Exactas, C. C. 67, \\ Universidad Nacional de La Plata, 1900 La Plata, Argentina \\ ${ }^{2}$ CIC nanoGUNE Consolider, Tolosa Hiribidea 76, 20018 Donostia, Spain \\ ${ }^{3}$ Laboratorio de Física del Sólido, Departamento de Física, FCEyT, Universidad Nacional de Tucumán, Tucumán, Argentina \\ ${ }^{4}$ Institute of Physics, Martin Luther University Halle-Wittenberg, Von-Seckendorff-Platz 1, 06120 Halle, Germany \\ ${ }^{5}$ Max Planck Institute of Microstructure Physics, Weinberg 2, 06120 Halle, Germany \\ ${ }^{6}$ Division of Superconductivity and Magnetism, Institute for Experimental Physics II, University of Leipzig, D-04103 Leipzig, Germany
}

(Received 20 June 2013; revised manuscript received 20 February 2014; published 13 March 2014)

\begin{abstract}
This work probes the relevance of oxygen vacancies in the formation of local ferromagnetic coupling between Fe ions at octahedral sites in zinc ferrites. This coupling gives rise to a ferrimagnetic ordering with the Curie temperatures above room temperature in an otherwise antiferromagnetic compound. This conclusion is based on experimental results from $\mathrm{x}$-ray magnetic circular dichroism measurements at the $\mathrm{Fe} \mathrm{L}_{2,3}$ edges and magnetization measurements performed on zinc ferrites, nanoparticles, and films, with different cation distributions and oxygen vacancy concentrations. Our observations are confirmed by density-functional-theory calculations and indicate that the enhanced ferrimagnetic response observed in some nominally nonmagnetic or antiferromagnetic ferrites can be taken as a further example of the defect-induced magnetism phenomenon.
\end{abstract}

DOI: 10.1103/PhysRevB.89.104411

PACS number(s): 78.70.Dm, 71.15.Mb, 75.47.Lx

\section{INTRODUCTION}

The observation of room-temperature ferromagnetism in nonmagnetic solids has been controversially related to the role of extrinsic or intrinsic defects [1-3]. Understanding the origin of the magnetic order and its coupling mechanism is essential for the development of new technological strategies to design materials suitable for spintronics applications. Extrinsic defects can be created by doping nonmagnetic oxide hosts with magnetic or nonmagnetic transition-metal ions, a research topic that has attracted considerable interest in recent decades [4]. On the other hand, the ferromagnetism displayed by some undoped nonmagnetic oxides has been linked to defects such as lattice vacancies or local disorder that is generated, for instance, by the reallocation of ions [5]. Both phenomena are known as defect-induced magnetism (DIM) [1-3]. The potential role of these defects in triggering or enhancing the ferromagnetic (FM) order in certain magnetic oxides has not yet been studied systematically.

One group of magnetic oxides, the spinel ferrites $M \mathrm{Fe}_{2} \mathrm{O}_{4}$ ( $M$ : divalent transition-metal cation), has been widely studied for years due to its diversity of ion configurations. Despite the relevance of the cationic distribution between the tetrahedral $\mathrm{A}$ and the octahedral B sites that determines the physical properties of spinels [6], there is still no consensus on the nature of the interactions responsible for the preferred groundstate structure [7,8]. Furthermore, the cation arrangement can be modified either by applying thermal treatments $[9,10]$, by using fabrication methods that make the formation of nonequilibrium phases easier [11-13], or by reducing the characteristic lengths of the specimen to the nanoscale [14].

\footnotetext{
*torres@ fisica.unlp.edu.ar

†stewart@fisica.unlp.edu.ar
}

It is well known that the disordered distribution of zinc and iron ions in $\mathrm{ZnFe}_{2} \mathrm{O}_{4}$ leads to a drastic change in its magnetic order. This phenomenon makes $\mathrm{ZnFe}_{2} \mathrm{O}_{4}$ one of the most studied ferrites in the area. Normal [15] $\mathrm{ZnFe}_{2} \mathrm{O}_{4}$ has an antiferromagnetic order below the Neél temperature $T_{\mathrm{N}}=10.5 \mathrm{~K}$, which is driven by an oxygen-mediated superexchange between the $\mathrm{Fe}^{3+}$ ions located at the B sites (case I in Fig. 1) [16]. However, some authors claim that $\mathrm{ZnFe}_{2} \mathrm{O}_{4}$ is intrinsically a frustrated system with a ferromagnetic first-neighbor exchange interaction $J_{\mathrm{BB}}>0$ (between two B sites) [17]. Anyway, all nanosized $\mathrm{ZnFe}_{2} \mathrm{O}_{4}$ samples, whether as nanoparticles (NPs) obtained by mechanical milling or by chemical routes [11,18], or as thin films prepared by sputtering [19] or by pulsed laser deposition (PLD) $[8,12,20]$, display ferrimagnetic behavior at room temperature. This ferrimagnetic feature is attributed to the distribution of $\mathrm{Fe}^{3+}$ and $\mathrm{Zn}^{2+}$ at both the $\mathrm{A}$ and $\mathrm{B}$ sites (case II in Fig. 1), which gives rise to strong negative $J_{\mathrm{AB}}$ interactions. The oxygen concentration also seems to be crucial for the cationic configuration since samples prepared under low oxygen pressure show a large magnetic response at room temperature $[17,21,22]$. It is likely that the disorder involving a cation swap is influenced by an oxygen displacement in the spinel structure $[7,22]$. The open question is whether the magnetization enhancement in $\mathrm{ZnFe}_{2} \mathrm{O}_{4}$ can be attributed only to a partial inversion, or also to local defects such as vacancies, or to interface effects in the case of thin films [23]. Since the knowledge of the structure of spinels at the microscopic level is still limited, the spin configuration and the origin of ferrimagnetism at room temperature in $\mathrm{ZnFe}_{2} \mathrm{O}_{4}$ remains unclear. Therefore, we use x-ray magnetic circular dichroism (XMCD) measurements and complementary ab initio densityfunctional-theory (DFT) calculations to prove that the enhanced magnetic response observed in nominally nonmagnetic or antiferromagnetic ferrites arises not only from cation inversion, but also from the existence of oxygen vacancies. 


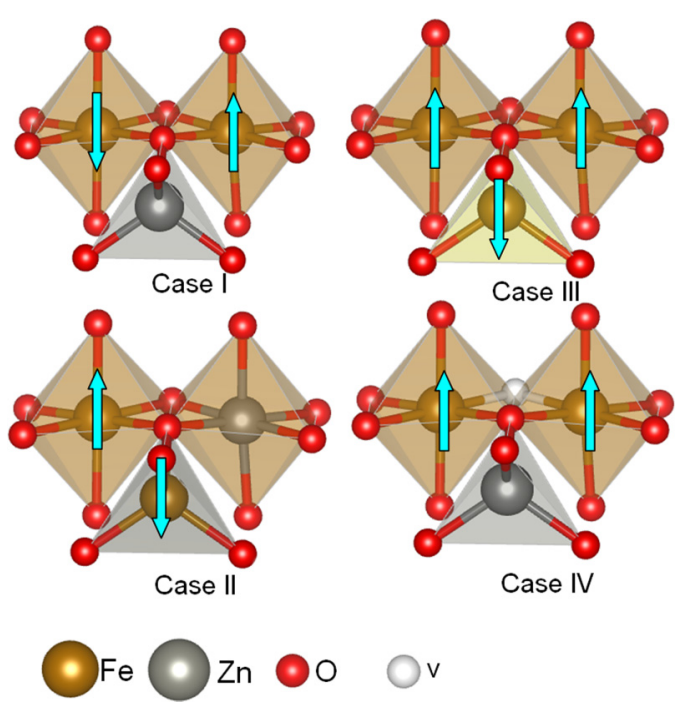

FIG. 1. (Color online) Sketched view of the possible magnetic configurations in zinc ferrites near tetrahedral (A sites) and octahedral (B sites) coordinated cations. The up and down arrows depict the moment alignment proposed for $\mathrm{Fe}^{3+}$ ions at each site. Case I: Normal state with $\mathrm{Zn}_{\mathrm{A}}$ and two $\mathrm{Fe}_{\mathrm{B}}$. Case II: Disordered state, where one $\mathrm{Fe}_{\mathrm{B}}$ migrates to the $\mathrm{A}$ site and the $\mathrm{Zn}_{\mathrm{A}}$ migrates to the $\mathrm{B}$ site. Case III: Iron occupying $\mathrm{A}$ and $\mathrm{B}$ sites, and zinc at otherwise unoccupied A sites (with respect to the normal spinel structure). Case IV: Iron occupying B sites, but mediated by an oxygen vacancy.

\section{EXPERIMENT}

\section{A. Sample description}

The investigated ferrites were (i) a thin film grown under low oxygen pressure conditions (ZFO1) of thickness $t=57 \mathrm{~nm}$, (ii) milled hydrothermally prepared $\mathrm{ZnFe}_{2} \mathrm{O}_{4}$ nanoparticles $(2 \mathrm{ZF} 10 \mathrm{H})$ with an average grain size of $D=13 \mathrm{~nm}$, and (iii) zinc ferrite nanoparticles obtained by mechanically grinding bulk $\mathrm{ZnFe}_{2} \mathrm{O}_{4}(\mathrm{BZF} 10 \mathrm{H})$ with $D=$ $14 \mathrm{~nm}$. The details of sample preparation and characterization can be found elsewhere [11,12]. All samples show a large saturation magnetization $M_{\mathrm{s}}$ (see Table I).

TABLE I. $M_{\mathrm{S}}$ is the macroscopic saturation magnetization measured with vibrating sample magnetometry (VSM) at $5 \mathrm{~K}$ with a maximum applied field $\mu_{0} H=5 \mathrm{~T} . I_{B_{2}} / I_{A}$ is the intensity ratio between the peaks $B_{2}$ and $A$ of the XMCD signal [see Fig. 2(b)]. $x_{\mathrm{B}} / x_{\mathrm{A}}$ is the relative $\mathrm{Fe}_{\mathrm{B}}^{3+}$ and $\mathrm{Fe}_{\mathrm{A}}^{3+}$ magnetic contribution ratio to the $\mathrm{XMCD}$ signal for each sample. $m_{\mathrm{XMCD}}$ and $m_{\mathrm{VSM}}$ are the magnetic moments per iron atom at room temperature determined either from the XMCD signals or $M_{\mathrm{s}}$ (measured by VSM), respectively.

\begin{tabular}{lccccc}
\hline \hline Sample & $\begin{array}{c}M_{\mathrm{s}} \\
\left(\mathrm{A} \mathrm{m}^{2} / \mathrm{kg}\right)\end{array}$ & $I_{B_{2}} / I_{A}$ & $x_{\mathrm{B}} / x_{\mathrm{A}}$ & $\begin{array}{c}m_{\mathrm{XMCD}} \\
\left(\mu_{\mathrm{B}} / \mathrm{Fe}\right)\end{array}$ & $\begin{array}{c}m_{\mathrm{VSM}} \\
\left(\mu_{\mathrm{B}} / \mathrm{Fe}\right)\end{array}$ \\
\hline $\mathrm{ZFO} 1$ & $360^{\mathrm{a}}$ & 3.3 & 2.2 & 0.93 & \\
$2 \mathrm{ZF} 10 \mathrm{H}$ & 68 & 2.3 & 1.9 & 0.59 & 0.58 \\
$\mathrm{BZF10H}$ & 72 & 2.2 & 1.9 & 0.57 & 0.57 \\
$\gamma-\mathrm{Fe}_{2} \mathrm{O}_{3} \mathrm{NPs}$ & & 1.5 & 1.6 & 0.80 & 0.84 \\
$\mathrm{Fe}_{3} \mathrm{O}_{4}$ bulk & & & & 1.20 & 1.20 \\
\hline \hline
\end{tabular}

${ }^{\mathrm{a}}$ For the film, the units are $\mathrm{A} / \mathrm{m}$.

\section{B. XMCD experiments}

XMCD experiments at the $\mathrm{L}_{2,3}$ edges of iron were performed at the PGM beam line at the Laboratorio Nacional de Luz Sincrotron, (LNLS, Campinas, Brazil), using an $80 \%$ circularly polarized light beam. The absorption data were collected at room temperature in total electron yield (TEY) mode. The samples were placed perpendicular to the photon beam and a magnetic field $\mu_{0} H_{\mathrm{ap}}=0.6 \mathrm{~T}$ was applied perpendicular to the sample plane. The absorption cross section of the circularly polarized $\mathrm{x}$ rays is labeled $\mu^{\alpha \beta}$, where $\alpha$ denotes the helicity of the photons $[\alpha=\uparrow(\downarrow)$ when the photons are right-hand (left-hand) polarized] and $\beta$ denotes the direction of $H_{\text {ap }}[\beta=\uparrow(\downarrow)$ when the field is parallel (antiparallel) to the beam direction]. The X-ray absorption spectra (XAS) are $\mu^{+}=\left(\mu^{\uparrow \uparrow}+\mu^{\downarrow \downarrow}\right) / 2$ and $\mu^{-}=\left(\mu^{\uparrow \downarrow}+\mu^{\downarrow \uparrow}\right) / 2$. The XMCD spectra were obtained as $\mu_{\mathrm{XMCD}}=\mu^{+}-\mu^{-}$.

\section{THEORY}

First-principles calculations have been performed with the Vienna $a b$ initio simulation package (VASP) code $[24,25]$ in order to prove that particular local arrangements (see Fig. 1) are energetically favorable and contribute ferromagnetically. The total-energy calculations are done in a cubic supercell configuration (including 56 atoms). The ground-state antiferromagnetic structure of Refs. [26,27] is used as a reference structure in our calculations. All calculations were performed with a plane-wave energy cutoff of $600 \mathrm{eV}$. For the Brillouinzone integration, we used a $\boldsymbol{\Gamma}$-centered Monkhorst-Pack $k$ space grid with $112 \boldsymbol{k}$ points. The exchange and correlation were treated by the generalized gradient approximation plus $U(\mathrm{GGA}+U)$ to describe the insulating and antiferromagnetic nature of $\mathrm{ZnFe}_{2} \mathrm{O}_{4}$ correctly. The correlation correction $(U=3.5 \mathrm{eV})$ is applied to the $d$ orbitals of iron. The minimumenergy lattice parameters obtained are $a=8.53 \AA$ and $u=0.381$, which are in good agreement with the experimental values and other calculations [28]. The positions of the ions in the supercell were relaxed by means of the conjugate gradient method until the forces on each atom converged to $7 \mathrm{meV} / \AA$.

Using this supercell, we studied the magnetic interactions under the influence of the local defects given in Fig. 1. The magnetic interaction is characterized by the energy difference between an antiparallel arrangement of the moments to that of a parallel moment arrangement on the corresponding atoms and is written as $\Delta E=E_{\mathrm{AFM}}-E_{\mathrm{FM}}$. A positive value of $\Delta E$ implies a local ferromagnetic (FM) order, while a negative value of $\Delta E$ demonstrates that antiferromagnetic (AFM) order is preferred. In order to validate our DFT calculation setup, we investigated the stability of the ideal antiferromagnetic (AFM) structure (case I in Fig. 1) with total-energy calculations. A local parallel arrangement of the magnetic moments of two $\mathrm{Fe}_{\mathrm{B}}^{3+}$ atoms leads to an increase of the energy by $\sim 32 \mathrm{meV}$ as compared to the AFM ground state and, therefore, is more unlikely (see Table II).

\section{RESULTS AND DISCUSSION}

The x-ray absorption spectra (XAS) of our three samples present the typical features [29] corresponding to the $2 p$ to $3 d$ electronic transitions [see Fig. 2(a) for ZFO1 film; the 
TABLE II. Total-energy difference $\Delta E=E_{\mathrm{AFM}}-E_{\mathrm{FM}}$ $\left(E_{\mathrm{AFM}}, E_{\mathrm{FM}}<0\right)$ and resulting magnetic moment $m$ from the theoretical calculations. The four cases correspond to the classification of Fig. 1. Case II is reached in a two-step model. S2 is the final result. See text for details.

\begin{tabular}{lcccc}
\hline \hline Property & Case I & Case II & Case III & Case IV \\
\hline$\Delta E(\mathrm{meV})$ & -31.80 & $-65.27(\mathrm{~S} 1)$ & 49.72 & 27.5 \\
& & $38.11(\mathrm{~S} 2)$ & & \\
$m\left(\mu_{\mathrm{B}} /\right.$ supercell $)$ & 0.0 & $0.0(\mathrm{~S} 1)$ & 6.0 & 8.0 \\
& & $10.0(\mathrm{~S} 2)$ & & \\
\hline \hline
\end{tabular}

spectra for $2 \mathrm{ZF} 10 \mathrm{H}$ and BZF10H are not shown]. For all of the studied samples, we obtained detailed information about the magnetic moment of the absorbing atom and its projection onto the direction of the applied field by XMCD measurements [see Fig. 2(b)]. For comparison purposes, we registered the XMCD spectrum of maghemite nanoparticles $\left(\gamma-\mathrm{Fe}_{2} \mathrm{O}_{3} \mathrm{NPs}\right)$ of a grain size $D=30 \mathrm{~nm}$, and of bulk magnetite $\left(\mathrm{Fe}_{3} \mathrm{O}_{4}\right)$ as well. Our results show that the XMCD signals of nanosized $\mathrm{Zn}$ ferrites at the $\mathrm{L}_{3}$ edge present one positive peak $A$ at about
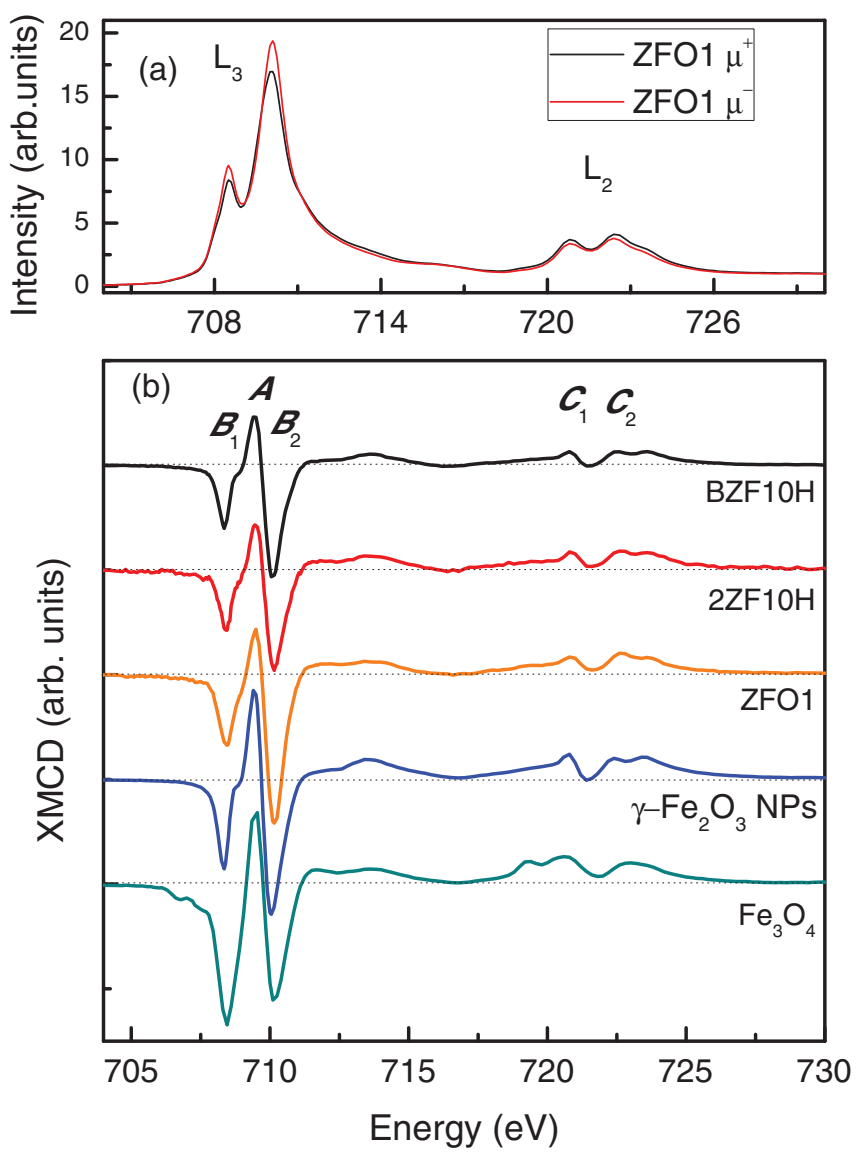

FIG. 2. (Color online) (a) Room-temperature XAS at the $\mathrm{L}_{2,3}$ edges of iron for sample ZF01 under a magnetic field of $0.6 \mathrm{~T}$, parallel $\mu^{+}$(and antiparallel $\mu^{-}$) to the propagation vector of a right-hand polarized photon. (b) XMCD signals of samples ZFO1, 2ZF10H, $\mathrm{BZF} 10 \mathrm{H}, \gamma-\mathrm{Fe}_{2} \mathrm{O}_{3} \mathrm{NPs}$, and bulk $\mathrm{Fe}_{3} \mathrm{O}_{4}$.
$709.5 \mathrm{eV}$ and two negative peaks $B_{1}$ and $B_{2}$ at $708.3 \mathrm{eV}$ and $710 \mathrm{eV}$, respectively [see Fig. 2(b)].

The XMCD spectra allow us to distinguish between the contributions of iron ions sitting either at $\mathrm{A}\left(\mathrm{Fe}_{\mathrm{A}}\right)$ or $\mathrm{B}\left(\mathrm{Fe}_{\mathrm{B}}\right)$ sites. In general, a negative XMCD signal at the $\mathrm{Fe} \mathrm{L}_{3}$ edge and a positive one at the $\mathrm{L}_{2}$ edge indicate that the iron magnetic moments are aligned parallel to the applied field. In inverse iron (III) ferrites such as maghemite, the iron ions $\mathrm{Fe}_{\mathrm{A}}^{3+}$ are antiferromagnetically coupled to the $\mathrm{Fe}_{\mathrm{B}}^{3+}$. This means, in an applied magnetic field, that the $\mathrm{Fe}_{\mathrm{B}}^{3+}$ moments align along the field direction, while the $\mathrm{Fe}_{\mathrm{A}}^{3+}$ ones orient in the opposite direction, leading to negative and positive dichroic signals at the $\mathrm{L}_{3}$ edge for $\mathrm{Fe}_{\mathrm{B}}^{3+}$ and $\mathrm{Fe}_{\mathrm{A}}^{3+}$, respectively. The opposite, i.e., a positive and negative peak (expressed as a small dip) for $\mathrm{Fe}_{\mathrm{B}}^{3+}$ and $\mathrm{Fe}_{\mathrm{A}}^{3+}$, respectively, is observed at the $\mathrm{L}_{2}$ edge. These two signals differ in shape and energy position. Thus, the resulting XMCD signal presents one positive peak corresponding mainly to $\mathrm{Fe}_{\mathrm{A}}^{3+}$ [labeled $A$ in Fig. 2(b)] and two negative peaks corresponding mainly to $\mathrm{Fe}_{\mathrm{B}}^{3+}$ [labeled $B_{1}$ and $B_{2}$ in Fig. 2(b)] [30-33]. On the other hand, the $L_{2}$ edge is characterized by two broad positive peaks of lower intensities [labeled $C_{1}$ and $C_{2}$ in Fig. 2(b)] and a dip in between [30-32].

The relative intensity between the $B_{2}$ and $A$ peaks qualitatively indicates the relative iron ions fraction at the $\mathrm{B}$ and $\mathrm{A}$ sites that contribute to the XMCD signal $\left(x_{\mathrm{B}} / x_{\mathrm{A}}\right)$. Comparing $\left(I_{B_{2}} / I_{A}\right)$ ratios for our three samples with each other, the ZFO1 film has the highest one [orange line in Fig. 2(b)]. This value is $44 \%$ larger for ZFO1 than those of the samples $2 \mathrm{ZF} 10 \mathrm{H}$ and BZF10H (see Table I). From this intensity ratio, we can estimate the relative magnetic contributions of the $\mathrm{Fe}_{\mathrm{B}}^{3+}$ and $\mathrm{Fe}_{\mathrm{A}}^{3+}$ to the XMCD signal $\left(x_{\mathrm{B}} / x_{\mathrm{A}}\right.$; see Refs. [30,34]). To this end, we have taken into account the results published by Brice-Profeta et al. [30]. These authors simulated, using ligand field multiplet calculations developed by De Groot et al. [34], the contribution of $\mathrm{Fe}^{3+}$ placed at $\mathrm{A}$ sites relative to $\mathrm{Fe}^{3+}$ placed at B sites. Using the simulated XMCD signals (at the $\mathrm{L}_{3}$ edge of $\left.\mathrm{Fe}\right)$ corresponding to $\mathrm{Fe}^{3+}$ placed at A sites $\left(T_{\mathrm{d}}\right.$ symmetry) and to $\mathrm{Fe}^{3+}$ placed at B sites ( $O_{\mathrm{h}}$ symmetry) (see bottom of Fig. 3 of Ref. [30]), we simulated the XMCD signals for different $x_{\mathrm{A}}$ and $x_{\mathrm{B}}$ values (see Fig. 3).

From these simulated signals, we extracted the values of the $I_{B_{2}} / I_{A}$ ratio for different $x_{\mathrm{B}} / x_{\mathrm{A}}$. Figure 4 shows the $x_{\mathrm{B}} / x_{\mathrm{A}}$

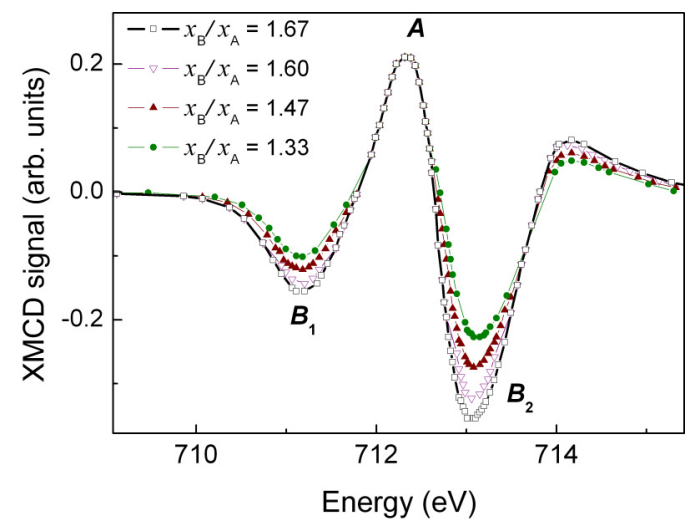

FIG. 3. (Color online) Simulation of XMCD signals for $\mathrm{Fe}_{\mathrm{B}}$ and $\mathrm{Fe}_{\mathrm{A}}$ with different relative magnetic contribution ratio $x_{\mathrm{B}} / x_{\mathrm{A}}$. 


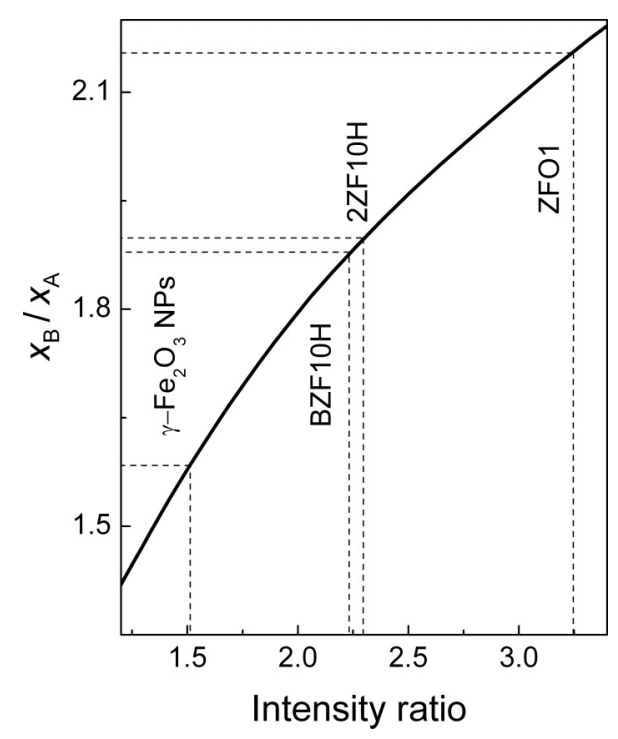

FIG. 4. Intensity ratio $I_{B_{2}} / I_{A}$ as a function of the ratio between iron ions at the $\mathrm{A}$ and $\mathrm{B}$ sites $\left(x_{\mathrm{B}} / x_{\mathrm{A}}\right)$ taken from Ref. [30]. The $\left(x_{\mathrm{B}} / x_{\mathrm{A}}\right)$ ratio for each sample was estimated considering the $I_{B_{2}} / I_{A}$ ratio taken from the experimental XMCD signals.

ratio versus $I_{B_{2}} / I_{A}$. Therefore, the $I_{B_{2}} / I_{A}$ ratios taken from our experimental results allow one to estimate the $x_{\mathrm{B}} / x_{\mathrm{A}}$ fraction for each sample (see Table I). Uncertainties of $I_{B_{2}} / I_{A}$ and $x_{\mathrm{B}} / x_{\mathrm{A}}$ are 0.05 and 0.02 , respectively. Again, for ZFO1, $x_{\mathrm{B}} / x_{\mathrm{A}}$ is higher than for the nanoparticles $2 \mathrm{ZF} 10 \mathrm{H}$ and BZF10H (see Table I). It is worth mentioning that the $x_{\mathrm{B}} / x_{\mathrm{A}}$ value obtained for $\gamma-\mathrm{Fe}_{2} \mathrm{O}_{3} \mathrm{NPs}$ is close to the $\mathrm{Fe}_{\mathrm{B}}^{3+} / \mathrm{Fe}_{\mathrm{A}}^{3+}$ ratio of about 1.67 expected for maghemite, since the whole $\mathrm{Fe}_{\mathrm{B}}^{3+}$ moments align along the field direction and the $\mathrm{Fe}_{\mathrm{A}}^{3+}$ ones orient in the opposite direction. From the XAS and XMCD spectra, we also estimate the total magnetic moment per iron atom, $m_{\mathrm{XMCD}}=m_{1}+m_{\mathrm{s}}$, with the orbital $\left(m_{1}\right)$ and spin $\left(m_{\mathrm{s}}\right)$ contributions, by using sum rules and integrating over the energy of the spectra [35]:

$$
\begin{gathered}
m_{1}=-\frac{2}{3} \frac{n_{\mathrm{h}}}{P_{\mathrm{c}} \cos \theta} \frac{\Delta W_{3}+\Delta W_{2}}{W_{3}+W_{2}}, \\
m_{\mathrm{s}}=-\frac{n_{\mathrm{h}}}{P_{\mathrm{c}} \cos \theta} \frac{\Delta W_{3}-2 \Delta W_{2}}{W_{3}+W_{2}}-\frac{7\left\langle T_{z}\right\rangle}{\left\langle S_{z}\right\rangle},
\end{gathered}
$$

where

$$
\begin{aligned}
& \Delta W_{i}=\int_{\mathrm{L}_{i}}\left(\mu^{+}-\mu^{-}\right) d E, \\
& W_{i}=\frac{1}{2} \int_{\mathrm{L}_{i}}\left(\mu^{+}+\mu^{-}\right) d E .
\end{aligned}
$$

$P_{\mathrm{c}}$ is the degree of circular polarization, $\theta$ is the angle between the $\mathrm{x}$-ray beam and the magnetization of the sample, $n_{\mathrm{h}}$ is the number of $d$ holes, and $\left\langle T_{z}\right\rangle$ and $\left\langle S_{z}\right\rangle$ are the expectation values of the magnetic dipole and spin component operators, respectively.

The total magnetic moment per iron atom is

$$
\begin{aligned}
m & =m_{1}+m_{\mathrm{s}} \\
& =-\frac{n_{\mathrm{h}}}{P_{\mathrm{c}} \cos \theta} \frac{1}{W_{3}+W_{2}}\left(\frac{5}{3} \Delta W_{3}-\frac{4}{3} \Delta W_{2}\right) .
\end{aligned}
$$

Here we have supposed that the relative dipolar contribution, i.e., $7\left\langle T_{z}\right\rangle /\left\langle S_{z}\right\rangle$, is negligible. This assumption is usually valid for ions having $d^{5}$ configuration in $O_{\mathrm{h}}$ or lower symmetries and $d^{6}$ in $O_{\mathrm{h}}$ symmetry $[36,37]$. Also, the fact that the XAS were registered at room temperature reduces its contribution [36].

XAS registered in TEY mode are typically affected by saturation effects [38], leading to an inaccurate quantitative analysis of $m_{1}$. Also, $m_{1}$ and $m_{\mathrm{s}}$ values are sensible to the normalization procedure and to the way the continuum spectrum is subtracted. Being aware of these problems, we have also determined $m$ for the reference samples $\gamma-\mathrm{Fe}_{2} \mathrm{O}_{3}$ NPs and bulk $\mathrm{Fe}_{3} \mathrm{O}_{4}$ (see Table I). The $m$ values obtained for the ferrites were divided by the $m$ value obtained for $\mathrm{Fe}_{3} \mathrm{O}_{4}$, and then multiplied by the moment of $1.20 \mu_{\mathrm{B}}$, as expected per iron atom for $\mathrm{Fe}_{3} \mathrm{O}_{4}$. In order to check the reliability of this procedure, we also applied this procedure to $\gamma-\mathrm{Fe}_{2} \mathrm{O}_{3} \mathrm{NPs}$ (Table I).

Also, the magnetic moments ( $\left.m_{\mathrm{VSM}}\right)$ were estimated from magnetization measurements at room temperature using a vibrating sample magnetometer (VSM). The average magnetic moment per iron atom for each powder sample was obtained dividing the total moment obtained at an applied magnetic field of $0.6 \mathrm{~T}$ (i.e., under the same condition as the XMCD measurements) by the number of magnetic atoms from saturation magnetization values. For all samples, both moments, $m_{\mathrm{XMCD}}$ and $m_{\mathrm{VSM}}$, agree very well (see Table I).

These experimental results for the magnetic moments and the ion distribution can be combined with two former investigations $[11,12]$ to discuss the local structure of our samples and the magnetic response in more detail. From the XMCD experiments, we confirmed that both $\mathrm{Fe}_{\mathrm{B}}^{3+}$ and $\mathrm{Fe}_{\mathrm{A}}^{3+}$ ions contribute to the dichroic signal. In particular, for the two nanoparticle samples $2 \mathrm{ZF} 10 \mathrm{H}$ and $\mathrm{BZF} 10 \mathrm{H}$, this quantitative contribution to the magnetic signal is $x_{\mathrm{B}} / x_{\mathrm{A}} \sim 2$. Since both samples are partially inverted ferrites (iron and zinc ions occupy both A and B sites; see Ref. [11]), they display the local magnetic configuration of case II (in Fig. 1). Furthermore, the same $x_{\mathrm{B}} / x_{\mathrm{A}}$ for $2 \mathrm{ZF} 10 \mathrm{H}$ and $\mathrm{BZF} 10 \mathrm{H}$ is consistent with their quite similar magnetic response, microstructure, and configuration of cations [11,39].

From the theoretical point of view, the AFM local rearrangement of case II also appeared energetically favorable [see Table II (S2)]. This local configuration was modeled in two steps. In the first step (S1), starting from the groundstate structure, a B-site $\mathrm{Fe}$ atom (represented as $\mathrm{Fe}_{2}$ in Fig. 5) was swapped with a $\mathrm{Zn}$ atom at an A site. A strong antiferromagnetic interaction is found between $\mathrm{Fe}_{1}$ and $\mathrm{Fe}_{2}$ because of the interaction $J_{\mathrm{AB}}$, which is strongly negative. In the second step (S2), keeping the alignment of moments of $\mathrm{Fe}_{1}$ and $\mathrm{Fe}_{2}$ fixed, the magnetic interaction between another $\mathrm{Fe}$ at a B site ( $\mathrm{Fe}_{3}$ in Fig. 5) and $\mathrm{Fe}_{1}$ is investigated. The parallel arrangement of the $\mathrm{Fe}_{1}$ and $\mathrm{Fe}_{3}$ moment is found to be lower in energy, implying $J_{\mathrm{BB}}>0$. Thus, we have a positive energy difference for $\mathrm{S} 2$ in Table II and this situation leads to local ferromagnetic order with $10 \mu_{\mathrm{B}}$ per supercell.

For the zinc ferrite film ZFO1, the XMCD results showed the same iron occupation at the A and $\mathrm{B}$ sites, with a larger contribution from the octahedral sites, characterized by markedly more intense $B_{1}$ and $B_{2}$ peaks [see Fig. 2(b)] and 


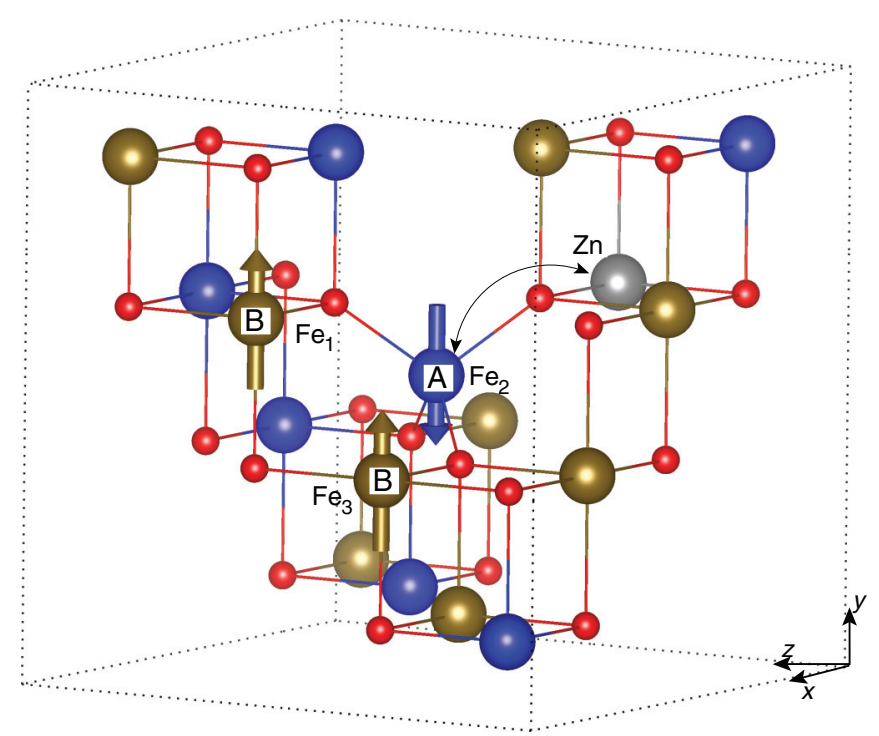

FIG. 5. (Color online) Figure depicting the situation of case II where the model is approached in two steps (see text). Rearrangement of atoms is achieved by swapping one Fe atom at the B site with that of one $\mathrm{Zn}$ atom at the $\mathrm{A}$ site (represented as black arrow). The most stable magnetic configuration with local ferromagnetic arrangement between $\mathrm{Fe}_{1}$ and $\mathrm{Fe}_{3}$ is shown, which were originally antiparallel in the ground-state cubic starting structure. $\mathrm{Zn}$ atoms are represented as gray, $\mathrm{O}$ as red, up-spin $\mathrm{Fe}$ as golden, and down-spin $\mathrm{Fe}$ as blue spheres, respectively.

the ratio $x_{\mathrm{B}} / x_{\mathrm{A}}>2$ (see Table $\mathrm{I}$ ). This detail complemented our former study [12], where the iron localization was not clearly detected from the XAS Fe $K$-edge results. In addition, from XAS Zn $K$-edge results for ZFO1, we have no indication of the presence of $\mathrm{Zn}^{2+}$ ions at the $\mathrm{B}$ sites [12]. So, $\mathrm{Fe}^{3+}$ ions migrate to the $\mathrm{A}$ sites, but the $\mathrm{Zn}^{2+}$ ions remain in their equilibrium location (A sites). Hence, we can conclude that instead of partial inversion (case II in Fig. 1), an unusual overpopulation of the A sites is present in the $\mathrm{ZnFe}_{2} \mathrm{O}_{4}$ film (in the normal spinel structure, only $1 / 8$ th of the $\mathrm{A}$ sites are occupied). This local configuration is depicted as case III (in Fig. 1). The calculation shows that an additional iron atom at an interstitial A site mediates and stabilizes a parallel arrangement of the moments of two $\mathrm{Fe}_{\mathrm{B}}$ ions together with large local relaxations. Therefore, they contribute to the ferrimagnetism with a moment of $6 \mu_{\mathrm{B}}$ per supercell (i.e., $10 \mu_{\mathrm{B}}$ per additional iron atom).

Furthermore, the magnetic moment per iron ion for the film ZFO1 (grown under low oxygen pressure) was $60 \%$ larger compared to the moment in the nanoparticles (see Table I). But, the probing depth of a few nanometers in the TEY mode at the L edges of iron rules out the possibility of having the magnetic ions located at the substrate-film interface and being the source of the unusually large B-site magnetic contribution in ZFO1. Furthermore, we know that among a set of ferrimagnetic zinc ferrite films, those grown under low oxygen pressure showed the highest magnetization [12]. So, we conclude that the low oxygen amount during film growth caused anion defects due to the incomplete oxidation of cations on each deposited layer (local configuration; see case IV in Fig. 1). Thus, it turns
TABLE III. Relaxation data near the oxygen vacancy, corresponding to case IV. Distances between bonding sites are given in \% change with respect to the ideal structure with bonds $\mathrm{Zn}-\mathrm{O}=2.0023 \AA$ and $\mathrm{Fe}-\mathrm{O}=2.0467 \AA$ (see Fig. 6 for the labels).

\begin{tabular}{lrcc}
\hline \hline Bonding sites & Rel. distance & Bonding sites & Rel. distance \\
\hline $\mathrm{Zn}_{1}-\mathrm{O}_{1}$ & 0.304 & $\mathrm{Fe}_{2}-\mathrm{V}_{\mathrm{O}}$ & 2.340 \\
$\mathrm{Zn}_{2}-\mathrm{V}_{\mathrm{O}}$ & 23.880 & $\mathrm{Fe}_{1}-\mathrm{O}_{2}$ & 1.162 \\
$\mathrm{Fe}_{1}-\mathrm{O}_{1}$ & -0.214 & $\mathrm{Fe}_{1}-\mathrm{O}_{2}^{\prime}$ & 3.692 \\
$\mathrm{Fe}_{1}-\mathrm{V}_{\mathrm{O}}$ & 0.970 & $\mathrm{Fe}_{2}-\mathrm{O}_{3}$ & 1.145 \\
$\mathrm{Fe}_{2}-\mathrm{O}_{1}$ & -2.733 & $\mathrm{Fe}_{2}-\mathrm{O}_{3}^{\prime}$ & -0.047 \\
\hline \hline
\end{tabular}

out that $\mathrm{ZnFe}_{2} \mathrm{O}_{4}$ is one of many compounds, where oxygen vacancies play an important role in the discussions of DIM [1-3,40,41].

In order to quantify the effects of a single vacancy as local defect in $\mathrm{ZnFe}_{2} \mathrm{O}_{4}$ and to complement the macroscopic experimental results, we investigated the local relaxation and the magnetic structure around this defect in more detail with the VASP code. The calculation supports the experimental conclusions that an oxygen vacancy between two $\mathrm{Fe}_{\mathrm{B}}^{3+}$ also mediates a FM coupling. The AFM coupling in the ideal AFM structure mediated through the $\mathrm{O}$ site, represented by a negative $J_{\mathrm{BB}}$ interaction, is now cut and the FM coupling of the two magnetic moments becomes more favorable, resulting in a magnetic moment of $8 \mu_{\mathrm{B}}$ per supercell (i.e., $8 \mu_{\mathrm{B}}$ per oxygen vacancy).

We compare the local changes in the lattice structure with the ideal AFM structure of case I (see Fig. 1), which corresponds to the perfect $\mathrm{ZnFe}_{2} \mathrm{O}_{4}$ in which all $\mathrm{Fe}-\mathrm{O}$ and $\mathrm{Zn}-\mathrm{O}$ bond lengths in the local environment are equivalent, as in the entire ferrite cell (see Table III). By introducing an oxygen vacancy, only the local surrounding as shown in Fig. 6 is affected. Due to the free space, the bond $\mathrm{Zn}_{1}$ $\mathrm{O}_{1}$ becomes elongated by about $0.3 \%$ (see Table III). The tetrahedral symmetry of the $\mathrm{Zn}$ atoms is broken and they show a tendency to form a dimer with the neighboring $\mathrm{Zn}$ atoms by moving away from $\mathrm{V}_{\mathrm{O}}$. So, the distance of $\mathrm{Zn}_{2}$ from the ideal $V_{O}$ position (the oxygen position inside an ideal

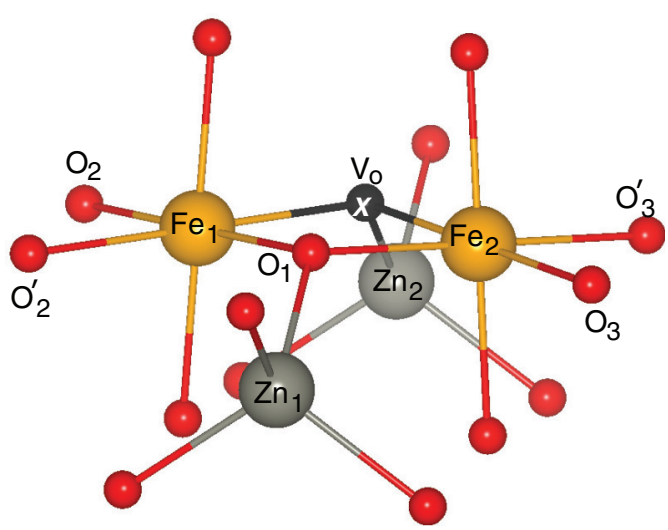

FIG. 6. (Color online) Local structure of $\mathrm{ZnFe}_{2} \mathrm{O}_{4}$ with labels used in Table III to explain the relaxation effect in the local environment of the oxygen vacancy [O (red), Fe site (golden), $\mathrm{Zn}$ site (gray), oxygen vacancy (black with white cross)]. 
(a)

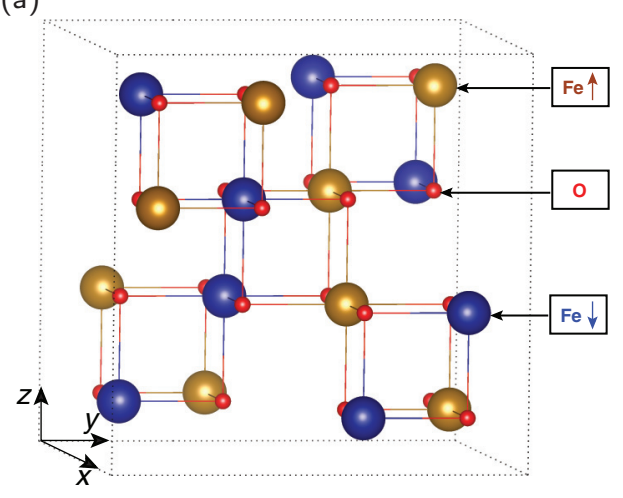

(b)

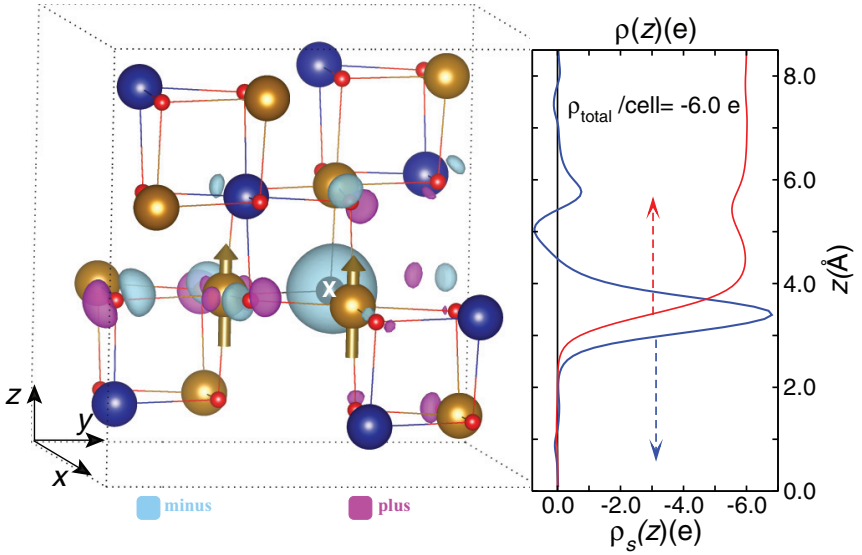

(c)

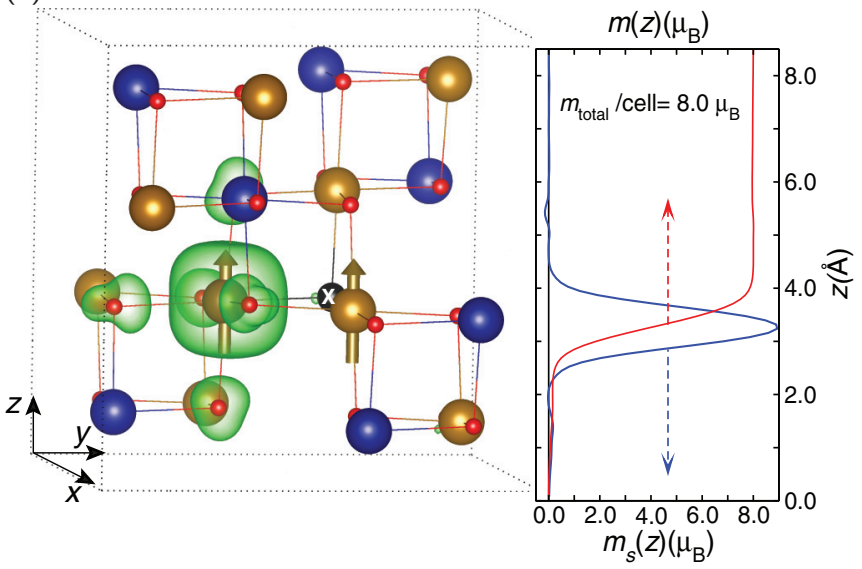

FIG. 7. (Color online) Theoretical results for the influence of an oxygen vacancy on the magnetic structure. (a) Supercell of the ideal (normal) structure of $\mathrm{ZnFe}_{2} \mathrm{O}_{4}$ (only the iron and oxygen atoms are shown). With respect to Fig. 5, the origin and the rotation of the cell are changed, which do not influence the results. Isosurfaces in the difference in (b) the charge density $\Delta \rho(\boldsymbol{r})$ and (c) the magnetization density $\Delta m(\boldsymbol{r})$ around an oxygen vacancy (sphere with white cross) with respect to the ideal structure in (a). The right-hand side graphs show those quantities integrated over the $x-y$ plane (blue line), i.e., in (b) $\rho_{s}(z)=\int \Delta \rho(\boldsymbol{r}) d s$, where the area element $d s=d x d y$, and additional integration along the $z$ direction, i.e., $\rho(z)=\int_{0}^{z} d z^{\prime} \rho_{s}\left(z^{\prime}\right)$ (red line). (c) Similar quantities, $m_{s}(z)$ (blue line) and $m(z)$ (red line), obtained from the difference of magnetization density.

structure) increases from 2.0 to $2.5 \AA$. In fact, performing cluster calculations for a free $\mathrm{Zn}_{2}$ dimer, we found that the free
Zn dimer has a bond length of $3.13 \AA$, which is comparable to $3.22 \AA$ of the $\mathrm{Zn}-\mathrm{Zn}$ separation in the neighborhood of $\mathrm{V}_{\mathrm{O}}$. The relaxation of the bond between $\mathrm{Zn}_{2}$ and its nearest $\mathrm{Zn}$ neighbor is $-12.7 \%$. In this case, a complete free dimer formation is hindered by other $\mathrm{Zn}-\mathrm{O}$ bonds associated with the $\mathrm{Zn}$ atoms.

$\mathrm{V}_{\mathrm{O}}$ is also responsible for an imbalance of bond lengths at the $\mathrm{Fe}_{1}$ and $\mathrm{Fe}_{2}$ sites. $\mathrm{Fe}_{2}-\mathrm{O}_{1}$ is elongated by about $2.0 \%$, while $\mathrm{Fe}_{1}-\mathrm{O}_{1}$ remains nearly unchanged (contracted by about $0.2 \%$ ). The three $\mathrm{Fe}$ atoms connected to $\mathrm{V}_{\mathrm{O}}$ are no longer equivalent and relax outwards with varying bond lengths of $1 \%$ and $2.3 \%$ for $\mathrm{Fe}_{1}-\mathrm{V}_{\mathrm{O}}$ and $\mathrm{Fe}_{2}-\mathrm{V}_{\mathrm{O}}$, respectively, and $2.5 \%$ for the $\mathrm{Fe}$ atom above $\mathrm{V}_{\mathrm{O}}$ (which is not shown). Away from the vacancy site, the bond lengths at Fe sites fluctuate between 0 and $4 \%$, which shows that the octahedral environment response to any defects in the tetrahedral region might be important. Furthermore, the calculations have been performed with different cell geometries leading to the same results.

In order to visualize the role of missing oxygen, the charge and magnetization densities in the supercell are calculated with an oxygen vacancy and compared to the ideal structure [shown in Fig. 7(a)]. The charge density changes abruptly at the vacancy site, but it also manifests a change in the oxygen atoms surrounding the iron ion that flips the moment; see Fig. 7(b). Due to this moment flip, the change in magnetization density reaches its highest value at the iron atom, while the oxygen atoms are also polarized and so contribute to the total moment of $8 \mu_{\mathrm{B}}$ per unit cell [see Fig. 7(c)]. Thus, the double $\mathrm{Fe}_{\mathrm{B}}^{3+}-\mathrm{O}^{-2}-\mathrm{Fe}_{\mathrm{B}}^{3+}$ superexchange paths are broken by the oxygen vacancies (case IV in Fig. 1) and contribute, therefore, to the $\mathrm{Fe}_{\mathrm{B}}-\mathrm{Fe}_{\mathrm{B}} \mathrm{FM}$ configuration, which is different from the AFM configuration displayed in normal ferrites (case I in Fig. 1).

\section{CONCLUSION}

We can conclude that even though samples ZFO1, 2ZF10H, and $\mathrm{BZF} 10 \mathrm{H}$ are all ferrimagnetic with relatively high saturation magnetizations, differences appear when exploring the distribution of cations that affect the local magnetic configuration. Our results demonstrate that the large magnetic moment observed in zinc ferrite thin films grown at low oxygen pressure is due to the ferromagnetic coupling between iron ions occupying octahedral B sites. The reason for this is based on three main mechanisms that break the original antiferromagnetic interaction between B sites, namely, the A-site overpopulation, the consequent generation of octahedral cation vacancies, and the existence of an oxygen vacancy between two $\mathrm{Fe}_{\mathrm{B}}$ ions.

So, the enhanced ferrimagnetic response observed in some nominally nonmagnetic or antiferromagnetic ferrites can be taken as a further example of the DIM phenomenon. By focusing on the defects-induced effects, our findings open up paths for applied research as well as attempts to further understand the nonequilibrium cation configuration, which gives rise to drastic changes in the magnetic behavior of certain ferrites.

\section{ACKNOWLEDGMENTS}

We appreciate the financial support from LNLS, Campinas, Sao Paulo, Brazil (proposals SGM and PGM 12490); 
CONICET (PIP 1111 and PIP 0324), Argentina; and from ANPCyT (PICT 1399-2008), Argentina. Part of this work has been financed by the DFG under the collaborative
Project No. SFB762 and MINCyT-DAAD cooperation Program No. DA13/02. We thank M. Ziese for providing sample ZF01.
[1] O. V. Yazyev, Rep. Prog. Phys. 73, 056501 (2010).

[2] O. Volnianska and P. Boguslawski, J. Phys. Condens. Matter 22, 073202 (2010).

[3] M. Stoneham, J. Phys. Condens. Matter 22, 074211 (2010).

[4] T. Dietl, Science 287, 1019 (2000).

[5] S. B. Ogale, Adv. Mater. 22, 3125 (2010).

[6] A. Goldman, in Modern Ferrite Technology, 2nd ed. (Springer, Boston, MA, 2006), p. 453.

[7] V. Stevanović, M. D’Avezac, and A. Zunger, J. Am. Chem. Soc. 133, 11649 (2011).

[8] V. Stevanović, M. d'Avezac, and A. Zunger, Phys. Rev. Lett. 105, 075501 (2010).

[9] H. S. C. O'Neill and A. Navrotsky, Amer. Mineralog. 68, 181 (1983).

[10] F. Bræstrup, B. C. Hauback, and K. K. Hansen, J. Solid State Chem. 181, 2364 (2008).

[11] S. J. Stewart, S. J. A. Figueroa, J. M. Ramallo López, S. G. Marchetti, J. F. Bengoa, R. J. Prado, and F. G. Requejo, Phys. Rev. B 75, 073408 (2007).

[12] C. E. Rodríguez Torres, F. Golmar, M. Ziese, P. Esquinazi, and S. P. Heluani, Phys. Rev. B 84, 064404 (2011).

[13] M. Bohra, S. Prasad, N. Kumar, D. S. Misra, S. C. Sahoo, N. Venkataramani, and R. Krishnan, Appl. Phys. Lett. 88, 262506 (2006).

[14] J. F. Hochepied, P. Bonville, and M. P. Pileni, J. Phys. Chem. B 104, 905 (2000).

[15] Normal refers to spinel ferrites with the eight divalent ions in the eight available tetrahedral sites and sixteen trivalent ions in the sixteen octahedral sites without antisite defects or vacancies.

[16] W. Schiessl, W. Potzel, H. Karzel, M. Steiner, G. M. Kalvius, A. Martin, M. K. Krause, I. Halevy, J. Gal, W. Schäfer, G. Will, M. Hillberg, and R. Wäppling, Phys. Rev. B 53, 9143 (1996).

[17] K. Kamazawa, Y. Tsunoda, H. Kadowaki, and K. Kohn, Phys. Rev. B 68, 024412 (2003).

[18] S. A. Oliver, H. H. Hamdeh, and J. C. Ho, Phys. Rev. B 60, 3400 (1999).

[19] S. Nakashima, K. Fujita, K. Tanaka, K. Hirao, T. Yamamoto, and I. Tanaka, Phys. Rev. B 75, 174443 (2007).

[20] Y. F. Chen, D. Spoddig, and M. Ziese, J. Phys. D 41, 205004 (2008).

[21] S. Ayyappan, S. P. Raja, C. Venkateswaran, J. Philip, and B. Raj, Appl. Phys. Lett. 96, 143106 (2010).
[22] J. Philip, G. Gnanaprakash, G. Panneerselvam, M. P. Antony, T. Jayakumar, and B. Raj, J. Appl. Phys. 102, 054305 (2007).

[23] M. N. Iliev, D. Mazumdar, J. X. Ma, A. Gupta, F. Rigato, and J. Fontcuberta, Phys. Rev. B 83, 014108 (2011).

[24] G. Kresse and J. Furthmüller, Comput. Mater. Sci. 6, 15 (1996).

[25] G. Kresse and J. Furthmüller, Phys. Rev. B 54, 11169 (1996).

[26] C. Cheng, Phys. Rev. B 78, 132403 (2008).

[27] C. Cheng and C.-S. Liu, J. Phys.: Conf. Ser. 145, 012028 (2009).

[28] S. Soliman, A. Elfalaky, G. H. Fecher, and C. Felser, Phys. Rev. B 83, 085205 (2011).

[29] J. P. Crocombette, M. Pollak, F. Jollet, N. Thromat, and M. Gautier-Soyer, Phys. Rev. B 52, 3143 (1995).

[30] S. Brice-Profeta, M.-A. Arrio, E. Tronc, N. Menguy, I. Letard, C. Cartier dit Moulin, M. Noguès, C. Chanéac, J.-P. Jolivet, and P. Sainctavit, J. Magn. Magn. Mater. 288, 354 (2005).

[31] R. A. D. Pattrick, G. Van Der Laan, C. M. B. Henderson, P. Kuiper, E. Dudzik, and D. J. Vaughan, Eur. J. Mineralog. 14, 1095 (2002).

[32] C. I. Pearce, Amer. Mineralog. 91, 880 (2006).

[33] B. T. Thole and G. van der Laan, Phys. Rev. B 38, 3158 (1988).

[34] F. M. F. de Groot, J. C. Fuggle, B. T. Thole, and G. A. Sawatzky, Phys. Rev. B 42, 5459 (1990).

[35] C. T. Chen, Y. U. Idzerda, H.-J. Lin, N. V. Smith, G. Meigs, E. Chaban, G. H. Ho, E. Pellegrin, and F. Sette, Phys. Rev. Lett. 75, 152 (1995).

[36] J. Crocombette, B. T. Thole, and F. Jollet, J. Phys. Condens. Matter 8, 4095 (1996).

[37] M. L. E. Goering, S. Gold, and G. Schütz, Europhys. Lett. 73, 97 (2006).

[38] R. Nakajima, J. Stöhr, and Y. U. Idzerda, Phys. Rev. B 59, 6421 (1999).

[39] S. J. Stewart, I. A. Al-Omari, F. R. Sives, and H. M. Widatallah, J. Alloys Comp. 495, 506 (2010).

[40] W. A. Adeagbo, G. Fischer, A. Ernst, and W. Hergert, J. Phys. Condens. Matter 22, 436002 (2010).

[41] M. Khalid, M. Ziese, A. Setzer, P. Esquinazi, M. Lorenz, H. Hochmuth, M. Grundmann, D. Spemann, T. Butz, G. Brauer, W. Anwand, G. Fischer, W. A. Adeagbo, W. Hergert, and A. Ernst, Phys. Rev. B 80, 035331 (2009). 